\title{
Crossing of the Phantom Divided Barrier with Lorentz Invariance Violating Fields
}

\author{
S. Davood Sadatian and Kourosh Nozari \\ Department of Physics, Faculty of Basic Sciences, \\ University of Mazandaran, \\ P. O. Box 47416-1467, Babolsar, IRAN \\ d.sadatian@umz.ac.ir, knozari@umz.ac.ir
}

\begin{abstract}
We study possible crossing of the phantom divided barrier in a Lorentz invariance violating dark energy model. Lorentz invariance violation which is achieved by introducing a vector field in the action, incorporates directly in the dynamics of the scalar field and equation of state. This interesting feature allows us to study phantom divided barrier crossing in the context of Lorentz invariance violation. We show that for suitable choice of parameter space, equation of state can cross phantom divided barrier just by one scalar field and Lorentz violating vector field controls this crossing.
\end{abstract}

PACS: 95.36.+x; 98.80.Cq; 98.80.-k

Key Words: Dark Energy Models, Scaler-Vector-Tensor Theories, Phantom Divided Barrier 


\section{Motivation}

Recently, Lorentz invariance violation (LIV) has been studied in the context of scalarvector-tensor theories[1]. It has been shown that Lorentz violating vector fields affect the dynamics of the inflationary models. One of the interesting feature of this scenario is that the exact Lorentz violating inflationary solutions are related to the absence of the inflaton potential. In this case, the inflation is completely associated with the Lorentz violation and depends on the value of the coupling parameter[2]. Standard cosmology with a pressureless fluid as matter content of the universe, predicts a universe either expanding forever or re-collapsing eventually depending on the spatial geometry. Recent evidences from supernova searches data [3,4], cosmic microwave background (CMB) results [5, 6, 7] and also Wilkinson Microwave Anisotropy Probe (WMAP) data [8, 9], indicate an accelerating phase of cosmological expansion today and this feature shows that the simple picture of universe consisting of pressureless fluid is not enough; the universe may contain some sort of additional negative-pressure dark energy. Analysis of the three year WMAP data $[10,11,12]$ shows that there is no indication for any significant deviations from Gaussianity and adiabaticity of the CMB power spectrum and therefore suggests that the universe is spatially flat to within the limits of observational accuracy. Further, the combined analysis of the three-year WMAP data with the supernova Legacy survey (SNLS)[10], constrains the equation of state $w_{d e}$, corresponding to almost $74 \%$ contribution of dark energy in the currently accelerating universe, to be very close to that of the cosmological constant value. Moreover, observations appear to favour a dark energy equation of state, $w_{d e}<-1$ [13]. Therefore a viable cosmological model should admit a dynamical equation of state that might have crossed the value $w_{d e}=-1$, in the recent epoch of cosmological evolution. Various aspects of this crossing has been studied extensively ( see for instance [14] and reference therein). However, possible impact between LIV and phantom divided

barrier crossing has not been studied yet. Since there are some traces of Lorentz invariance violation in high-energy regime $[15,16]$, it is interesting to study possible implication of this symmetry breaking on the dynamics of equation of state and especially crossing of the phantom divide barrier. The purpose of this letter is to take a small step in this direction.

\section{A Lorentz Violating Cosmology}

In this section, following $[1,2]$, we summarize the cosmological dynamics of Lorentz invariance violating fields. Our goal is to find a relation between Lorentz Invariance violation 
parameter and dynamics of scalar field. This relation will affect the equation of state of scalar field which is the central object of subsequent sections.

We start with the following action for a typical scalar-vector-tensor theory which admits Lorentz invariance violation

$$
S=S_{g}+S_{u}+S_{\phi}
$$

where the actions for the tensor field $S_{g}$, the vector field $S_{u}$, and the scalar field $S_{\phi}$ are defined as follows

$$
\begin{aligned}
S_{g}= & \int d^{4} x \sqrt{-g} \frac{1}{16 \pi G} R \\
S_{u}= & \int d^{4} x \sqrt{-g}\left[-\beta_{1} \nabla^{\mu} u^{\nu} \nabla_{\mu} u_{\nu}-\beta_{2} \nabla^{\mu} u^{\nu} \nabla_{\nu} u_{\mu}-\beta_{3}\left(\nabla_{\mu} u^{\mu}\right)^{2}\right. \\
& \left.-\beta_{4} u^{\mu} u^{\nu} \nabla_{\mu} u^{\alpha} \nabla_{\nu} u_{\alpha}+\lambda\left(u^{\mu} u_{\mu}+1\right)\right], \\
S_{\phi}= & \int d^{4} x \sqrt{-g} \mathcal{L}_{\phi} .
\end{aligned}
$$

This action is allowed to contain any non-gravitational degrees of freedom in the framework of Lorentz violating scalar-tensor-vector theory of gravity. As usual, we assume $u^{\mu} u_{\mu}=-1$ and that the expectation value of vector field $u^{\mu}$ is $<0\left|u^{\mu} u_{\mu}\right| 0>=-1[17]$. $\beta_{i}(\phi)(i=1,2,3,4)$ are arbitrary parameters with dimension of mass squared and $\mathcal{L}_{\phi}$ is the Lagrangian density for scalar field. Note that $\sqrt{\beta_{i}}$ are mass scale of Lorentz symmetry breakdown $[1,17]$. The detailed cosmological consequences of this action are studied in Ref.[1].

Assuming a homogeneous and isotropic universe, we describe the universe with the following metric

$$
d s^{2}=-\mathcal{N}^{2}(t) d t^{2}+e^{2 \alpha(t)} \delta_{i j} d x^{i} d x^{j}
$$

where $\mathcal{N}$ is a lapse function and the scale of the universe is determined by $\alpha[1,2]$. By variation of the action with respect to metric and choosing a suitable gauge, one obtains the following field equations

$$
R_{\mu \nu}-\frac{1}{2} g_{\mu \nu} R=8 \pi G T_{\mu \nu}
$$

where $T_{\mu \nu}=T_{\mu \nu}^{(u)}+T_{\mu \nu}^{(\phi)}$ is the total energy-momentum tensor, $T_{\mu \nu}^{(u)}$ and $T_{\mu \nu}^{(\phi)}$ are the energy-momentum tensors of vector and scalar fields, respectively. The time and space components of the total energy-momentum tensor are given by[2]

$$
T_{0}^{0}=-\rho_{u}-\rho_{\phi}, \quad T_{i}^{i}=p_{u}+p_{\phi}
$$


where the energy density and pressure of the vector field are calculated as follows

$$
\begin{aligned}
& \rho_{u}=-3 \beta H^{2}, \\
& p_{u}=\left(3+2 \frac{H^{\prime}}{H}+2 \frac{\beta^{\prime}}{\beta}\right) \beta H^{2}, \\
& \beta \equiv \beta_{1}+3 \beta_{2}+\beta_{3},
\end{aligned}
$$

a prime denotes the derivative of any quantity with respect to $\alpha$ and $H \equiv d \alpha / d t=\dot{\alpha}$ is the Hubble parameter. One can see that $\beta_{4}$ does not contribute to the background dynamics[1,2]. The energy equations for the vector field $u$ and scalar field, $\phi$ are as follows

$$
\begin{aligned}
& \rho_{u}^{\prime}+3\left(\rho_{u}+p_{u}\right)=+3 H^{2} \beta^{\prime}, \\
& \rho_{\phi}^{\prime}+3\left(\rho_{\phi}+p_{\phi}\right)=-3 H^{2} \beta^{\prime},
\end{aligned}
$$

respectively. So, the total energy equation in the presence of both the vector and the scalar fields reads

$$
\rho^{\prime}+3(\rho+p)=0, \quad\left(\rho=\rho_{u}+\rho_{\phi}\right) .
$$

With these preliminaries, dynamics of the model is described by the following Friedmann equations $[1,2]$

$$
\begin{array}{r}
\left(1+\frac{1}{8 \pi G \beta}\right) H^{2}=\frac{1}{3 \beta} \rho_{\phi}, \\
\left(1+\frac{1}{8 \pi G \beta}\right)\left(H H^{\prime}+H^{2}\right)=-\frac{1}{6}\left(\frac{\rho_{\phi}}{\beta}+\frac{3 p_{\phi}}{\beta}\right)-H^{2} \frac{\beta^{\prime}}{\beta} .
\end{array}
$$

In the absence of vector field, that is, when all $\beta_{i}=0$, one recovers the standard equations of dynamics. For the scalar sector of our model we assume the following Lagrangian

$$
\mathcal{L}_{\phi}=-\frac{\eta}{2}(\nabla \phi)^{2}-V(\phi),
$$

where $(\nabla \phi)^{2}=g^{\mu \nu} \partial_{\mu} \phi \partial_{\nu} \phi$. Ordinary scalar fields are correspond to $\eta=1$ while $\eta=-1$ describes phantom fields. For the homogeneous scalar field, the density $\rho_{\phi}$ and pressure $p_{\phi}$ are given as follows

$$
\begin{aligned}
& \rho_{\phi}=\frac{\eta}{2} H^{2} \phi^{2}+V(\phi), \\
& p_{\phi}=\frac{\eta}{2} H^{2} \phi^{\prime 2}-V(\phi) .
\end{aligned}
$$


The corresponding equation of state parameter is

$$
\omega_{\phi}=\frac{p_{\phi}}{\rho_{\phi}}=-\frac{1-\eta H^{2} \phi^{2} / 2 V}{1+\eta H^{2} \phi^{2} / 2 V} .
$$

Now the Friedmann equation takes the following form [2]

$$
H^{2}=\frac{1}{3 \bar{\beta}}\left[\frac{\eta}{2} H^{2} \phi^{\prime 2}+V(\phi)\right]
$$

where $\bar{\beta}=\beta+\frac{1}{8 \pi G}$. Using this equation we can show that

$$
\phi^{\prime}=-2 \eta \bar{\beta}\left(\frac{H_{, \phi}}{H}+\frac{\bar{\beta}_{, \phi}}{\bar{\beta}}\right) .
$$

Substituting this equation into the Friedmann equation, the potential of the scalar field can be written as [2]

$$
V=3 \bar{\beta} H^{2}\left[1-\frac{2}{3} \eta \bar{\beta}\left(\frac{\bar{\beta}_{, \phi}}{\bar{\beta}}+\frac{H_{, \phi}}{H}\right)^{2}\right]
$$

Note that in the above equations the Hubble parameter $H$ has been expressed as a function of $\phi, H=H(\phi(t))$. One can show that the equation of state has the following form

$$
\begin{aligned}
\omega_{\phi} & =-1+\frac{4}{3} \eta \bar{\beta}\left(\frac{H_{, \phi}}{H}+\frac{\bar{\beta}_{, \phi}}{\bar{\beta}}\right)^{2} \\
& =-1+\frac{1}{3} \eta \frac{\phi^{\prime 2}}{\bar{\beta}} .
\end{aligned}
$$

Equations (21) and (23) are essential in forthcoming discussions. Note that violation of the Lorentz invariance which has been introduced by existence of a vector field in the action, now has incorporated in the dynamics of scalar field and equation of state via existence of $\bar{\beta}$. This interesting feature allows us to study phantom divided barrier crossing in the context of Lorentz invariance violation. We need to solve these two equations, (21) and (23), to find dynamics of scalar field $\phi$ and the equation of state $\omega_{\phi}$. This will be achieved only if the Hubble parameter $H(\phi)$ and the vector field coupling, $\bar{\beta}(\phi)$ are known. In which follows, our strategy is to choose some different cases of the Hubble parameter $H(\phi)$ and the vector field coupling $\bar{\beta}(\phi)$ and then investigating possible crossing of phantom divided barrier in this context. We concentrate on suitable domains of parameter space which admit such a crossing. 


\section{LIV and Crossing of the Phantom Divided Barrier}

To investigate phantom divided barrier crossing in this context, we have to solve equations (21) and (23) for four unknowns: $H, \omega_{\phi}, \bar{\beta}$, and $V$. This is impossible unless two of these unknowns be specified a priori. A large class of equations of state for scalar field has been studied in [18] and [19] and some classes of potentials allowing for the scalar field equation of state were described. Also some authors have used vector field models for coincidence of dark energy $[20,21,22,23]$. The main point of these studies which is crucial for our subsequent discussions is the fact that to have a successful model with phantom divided barrier crossing within a minimally coupled scalar field scenario, one should consider both quintessence and phantom fields. However, with non-minimally coupled scalar fields, one can achieve phantom divided barrier crossing just with one of these fields[14]. As we will show, in the presence of Lorentz invariance violating fields, it is possible to cross phantom divided barrier just by considering one field, i. e., quintessence field. This may reflect the fact that LIV has something to do with non-minimal coupling of scalar field and gravity. In other words, our basic action defined in equations (1) and (2) are actually minimally coupled, but our results for crossing of phantom divide barrier are very similar to non-minimal model results presented in [14]. So, it seems that there is a close relation between LIV and non-minimal scenario in the present context. In which follows, based on above argument, we consider just a quintessence scalar field with $\eta=1$. We study possible crossing of the phantom divided barrier in some model universes with concentration on suitable range of the parameter space.

\subsection{Case 1}

In the first step we consider a model universe with the following simple choices

$$
H=H_{0}, \quad \bar{\beta}(\phi)=m \phi^{2},
$$

where $H_{0}$ is a positive constant. With these choices, equations (21) and (23) lead us to the following relations

$$
\begin{gathered}
\phi(t)=\phi_{0} \exp \left[-4 \eta m H_{0}\left(t-t_{0}\right)\right], \\
\omega_{\phi}=-1+\frac{16}{3} m,
\end{gathered}
$$

where $\phi\left(t=t_{0}\right) \equiv \phi_{0}$ is a constant. This model shows that cosmic evolution starts

from a constant value of the scale factor and then grows exponentially, $a(t)=a_{0} e^{H_{0}\left(t-t_{0}\right)}$. 
Equation (26) shows that the equation of state $\omega_{\phi}$ has no dynamics since it only depends on the value of the vector field coupling parameter $m$. Since an accelerated expansion occurs for $\omega_{\phi}<-1 / 3$ [2], then we should have $m<1 / 8$ for a typical quintessence field. However, the present data of the universe seems to tell us that $\omega_{\phi}$ might be less than -1 . Thus, the value of $m$ may be chosen to fit with the present observable constraint on the equation of state parameter. If we use the values of $m$ obtained in [2], we can calculate $\omega_{\phi}$ versus $m$. This is shown in figure 1 . This figure shows that for crossing of

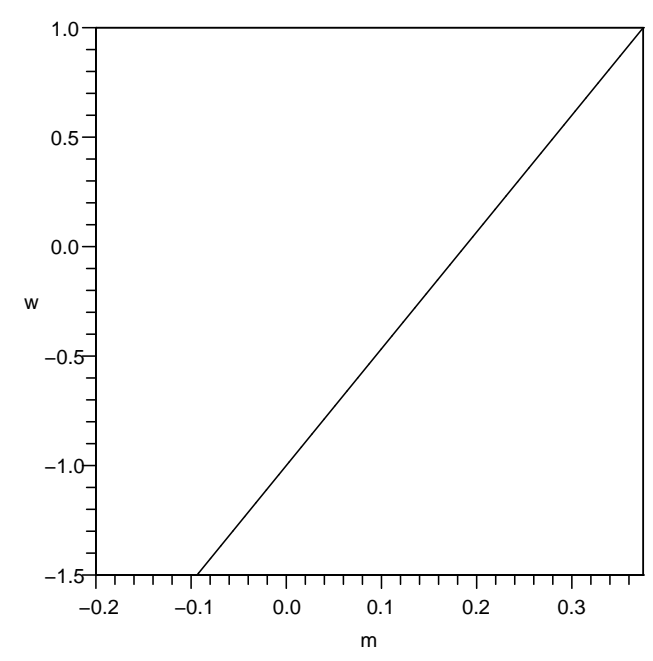

Figure 1: Variation of $\omega_{\phi}$ for different values of the coupling vector parameter $m$.

phantom divided line, $\omega_{\phi}=-1$, the lower value of $m$ should be negative. However this is impossible since $m$ is related to $\sqrt{\beta_{i}}$ as mass scales of Lorentz symmetry breakdown. So, in this model universe, crossing of phantom divided barrier with one scalar field is impossible. This is not surprising since equation of state in this case has no dynamics and there is no reasonable fine tuning. However it is simple to show that existence of two scalar fields, one quintessence and the other phantom field, as usual can lead to possibility of cosmological constant equation of state crossing. Note that in Ref.[2], the authors have found that $m$ should be restricted to interval $1 / 6<m<3 / 8$ to provide a suitable inflation model. In summary, this model universe provides no consistent scenario for a dynamical equation of state containing phantom divided barrier crossing. 


\section{$3.2 \quad$ Case 2}

As a second case, we suppose $H(\phi)=H_{0} \phi^{\xi}$ and $\bar{\beta}(\phi)=m \phi^{2}$. With these choices, we find the following equation of state

$$
\omega_{\phi}=-1+\frac{4}{3} m(\xi+2)^{2} .
$$

which evidently has no dynamics. The condition for acceleration of the universe, $H^{\prime} / H>$ -1 , yields

$$
m<\frac{1}{2 \xi(\xi+2)}
$$

The scalar field evolve as follows

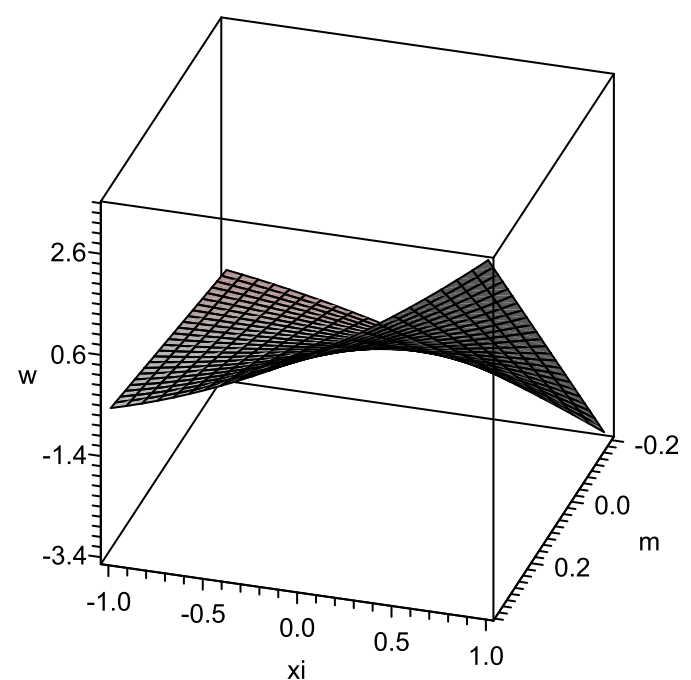

Figure 2: Variation of $\omega_{\phi}$ for different values of the vector field coupling parameter $m$ and $\xi$. Note that based on relation (28), if we choose $-1<\xi<1$ then $m$ should be restricted to the interval $-0.5<m<0.166$. However $m$ can not attain negative values and therefore we should restrict this interval to $0<m<0.166$.

$$
\phi(t)=\phi_{0}\left(1+\frac{H_{0} \phi_{0}^{\xi}}{p}\left(t-t_{0}\right)\right)^{-1 / \xi} .
$$

The variation of $\omega_{\phi}$ versus $m$ and $\xi$ is shown in figure 2. This figure shows that only for positive values of $m$ and all possible values of $\xi$, the equation of state satisfies the condition $\omega_{\phi}>-1$. For other positive values of $\xi$ and negative values of $m$, equation of state has a phantom divide line since $\omega_{\phi}<-1$. But we should stress that since $m$ cannot attain negative values, we must restrict $m$ to the interval $0<m<0.166$. As figure shows, 
with this restriction it is impossible to cross phantom divided line with one scalar field.

In these two examples, the equation of state has no dynamics. Only with variation of parameters $m$ and $\xi$ one can formally attain a phantom divided barrier crossing. The case with a dynamical equation of state is more reasonable. Now we turn to the situation where the equation of state is dynamical. For this purpose, we generalize the vector field coupling to achieve the value of $\bar{\beta}(\phi)=m \phi^{n}$, for $n>2$.

\subsection{Case 3}

To have a dynamical equation of state, we consider a model universe where the vector field coupling parameter is written as a power of the scalar field

$$
H=H_{0}, \quad \bar{\beta}(\phi)=m \phi^{n}, \quad n>2,
$$

where $H_{0}$ and $n$ are constant positive parameters. Following the above procedure, the scalar field $\phi$, the vector field coupling $\bar{\beta}$ and equation of state $\omega_{\phi}$ have the following dynamics[2]

$$
\begin{aligned}
\phi(t) & =\frac{\phi_{0}}{\left[1+2 m n H_{0}(n-2) \phi_{0}^{n-2}\left(t-t_{0}\right)\right]^{\frac{1}{n-2}}}, \\
\bar{\beta}(t) & =\frac{m \phi_{0}^{n}}{\left[1+2 m n H_{0}(n-2) \phi_{0}^{n-2}\left(t-t_{0}\right)\right]^{\frac{n}{n-2}}}, \\
\omega_{\phi}(t) & =-1+\frac{4 m n^{2} \phi_{0}^{n-2} / 3}{1+2 m n H_{0}(n-2) \phi_{0}^{n-2}\left(t-t_{0}\right)} .
\end{aligned}
$$

Remember that $\bar{\beta}(t)$ plays the role of Lorentz invariance violation in this setup. Equation of dynamics for $\bar{\beta}(t)$ implicitly has an important meaning: by a suitable fine tuning one can construct a Lorentz violating cosmology consistent with observational data. In another words, this setup provides an important basis for testing LIV in cosmological context.

Although many different models can also lead to phantom divided barrier crossing, our model is special in this respect since it contains only one scalar field and the presence of Lorentz violating vector field controls the crossing. In this sense, fine tuning of parameters space based on observational data restricts the value that $\bar{\beta}(t)$ can attain. Any nonvanishing value of $\bar{\beta}$ in our model shows violation of Lorentz symmetry in this cosmological setup. If dynamics of $\bar{\beta}(t)$ which is described by equation (32) can be detected and 
constraint by observational data, this will be a manifestation of LIV in cosmological context. Lorentz invariance violating inflation models constraint by WMAP and other observational data my provide other test of LIV in cosmological setup. To see possible detection of Lorentz-violating field in cosmology see $[25,28,29]$.

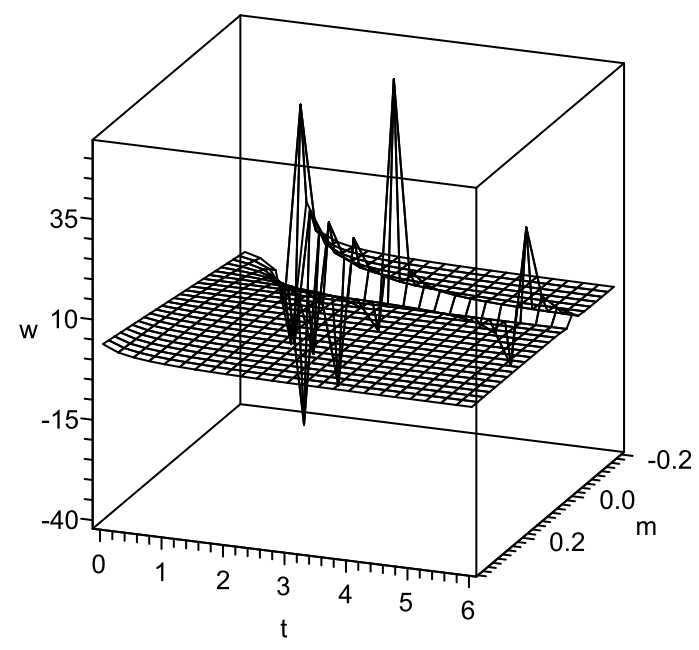

Figure 3: Variation of $\omega_{\phi}$ for different values of the vector field coupling $m$ and $t$ for $n=3$.

In this model, the cosmic evolution starts from a constant value of the scale factor and then grows exponentially, $a(t)=a_{0} e^{H_{0}\left(t-t_{0}\right)}$, while the vector field coupling, $\bar{\beta}$, starts from a constant value of the scalar field, $m \phi_{0}^{n}$. The equation of state $\omega_{\phi}$ is dynamical. Figure 3 shows the variation of the equation of state versus $m$ and $t$. This figure clearly demonstrates a crossing of the phantom divided line. We stress that such a results are essentially model dependent. In principle, these results should be translated to red-shift language which provides a better framework to compare with observational data. While this is essentially important, the purpose of this letter is to show that LIV may help us to achieve phantom divided line crossing with only one scalar field. In other words in the presence of LIV, just one scalar field is enough to achieve phantom divided barrier crossing and existence of vector field controls the situation.

\subsection{A more General case}

In this section we consider a more general case where both the vector field coupling and the Hubble parameter are functions of $\phi$ defined as follows

$$
H=H_{0} \phi^{\xi}, \quad \bar{\beta}(\phi)=m \phi^{n}, \quad n>2
$$


Using equation (21), for this case we obtain

$$
\phi(t)=\frac{1}{\left[H_{0}\left(t-t_{0}\right)\left(-4 \xi m+4 \xi m n+2 \xi^{2} m-4 m n+2 m n^{2}\right)+\phi_{0}\right]^{\left(\frac{1}{n+\xi-2}\right)}}
$$

and using equation (23) we find

$$
\omega_{\phi}(t)=-1+\frac{4}{3} m \phi^{n-2}(t)(\xi+n)^{2}
$$

The condition for acceleration of the universe, that is, $H^{\prime} / H>-1$, yields

$$
m^{2}<\frac{1}{4(-1)^{n} \phi^{n-2}(t)(\xi+n)^{2}}, \quad n>2,
$$

With $\phi$ defined as (35), the equation of state takes the following form

$$
\omega_{\phi}(t)=-1+\frac{4}{3} m \frac{(\xi+n)^{2}}{\left[H_{0}\left(t-t_{0}\right)\left(-4 \xi m+4 \xi m n+2 \xi^{2} m-4 m n+2 m n^{2}\right)+\phi_{0}\right]^{\left(\frac{n-2}{n+\xi-2}\right)}},
$$

which explicitly has a dynamical behavior. This model allows us to choose a suitable parameter space to cross phantom divided barrier. This parameter space should be checked by observational data to have a reasonable model. The most important aspect of the present model is the fact that in principle, LIV fields provide situation that one scalar field and another vector field together can lead us to describe phantom divided barrier crossing.

Figure 4 shows the crossing of phantom divided barrier for a dynamical equation of state. Figure 4 may be used to explain why we are living in an epoch of $\omega<-1$ since in late time we see that $\omega<-1$. This is the second cosmological coincidence problem. Two point should be stressed here: firstly, as figures 3 and 4 show, there are some sudden jumps of the equation of state. In many existing models whose equation of state can cross the phantom divided barrier, $\omega$ undulates around -1 randomly ([24] and references therein). These jumps are actually a manifestation of this undulation which may be a signature of chaotic behavior of equation of state. Secondly, as these figures show, crossing of the phantom divided barrier can occur at late-time. This fact, as second cosmological coincidence problem, needs additional fine-tuning in model parameters and trigger mechanism, for instance, can be used to alleviate this coincidence. 


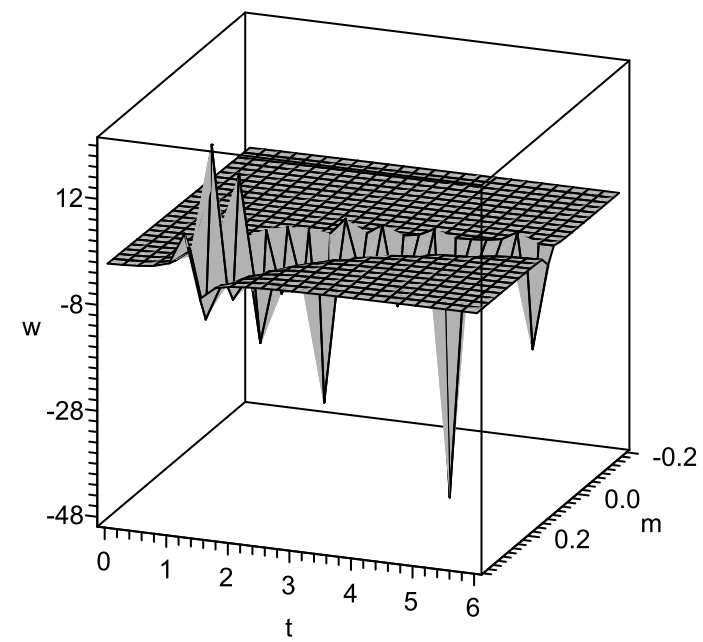

Figure 4: Variation of $\omega_{\phi}$ for different values of the vector field coupling $m$ and $t$ for $n=3$ and $\xi=-2$. Positive values of $\xi$ show no phantom divided barrier crossing. The values of $\xi$ are determined by relation (37).

\section{Conclusions}

As a new mechanism for crossing of phantom divided barrier by equation of state, in this paper we have incorporated a possible violation of Lorentz invariance in a cosmological setup. We have shown that by a suitable choice of parameter space, it is possible to have phantom divided barrier crossing in a Lorentz invariance violating context just by a single scalar field. This is an important result since in the absence of LIV, as previous studies have shown, it is impossible to cross phantom divided barrier by just one scalar field minimally coupled to gravity[14,27]. In this regard, existence of a Lorentz invariance violating vector field provides a framework for crossing phantom divided barrier with one scalar field. On the other hand, this model provides a possible framework for testing Lorentz invariance violation in a cosmological context. Using observational data and by a suitable fine tuning it is possible to construct a reliable Lorentz violating cosmological model. A similar strategy has been applied for inflation in reference[2]. Another aspect of our model is the fact that it contains several crossing of phantom divided barrier, a phenomena which has been seen in other scenarios[30,31,32]. As we have shown, the equation of state takes different form in different choices of parameter space.

In this framework it is possible to use the "trigger mechanism" to explain dynamical equation of state. This means that we assume scalar- vector-tensor theory containing Lorentz invariance violation which acts like the hybrid inflation models. In this situation, 
vector and scaler field play the roles of inflaton and the "waterfall" field respectively. This conjecture is under investigation[26].

\section{References}

[1] S. Kanno and J. Soda, Phys. Rev. D 74 (2006) 063505

[2] Arianto, Freddy P. Zen, Bobby E. Gunara, Triyanta and Supardi, JHEP 09 (2007) 048

[3] S. Perlmutter, et al, Astrophys. J. 517 (1999) 565

[4] A. G. Riess, et al, Astron. J. 116 (1998) 1009

[5] A. D. Miller, et al, Astrophys. J. Lett. 524 (1999) L1

[6] P. de Bernardis, et al, Nature 404 (2000) 955

[7] S. Hanany, et al, Astrophys. J. Lett. 545 (2000) L5

[8] D. N. Spergel, et al, Astrophys. J. Suppl. 148 (2003) 175

[9] L. Page, et al, Astrophys. J. Suppl. 148 (2003) 233

[10] D. N. Spergel, et al, Astrophys. J. Suppl. 170 (2007) 377

[11] G. Hinshaw, et al, Astrophys. J. Suppl. 170 (2007) 288

[12] L. Page, et al, Astrophys. J. Suppl. 170 (2007) 335

[13] A. G. Reiss, et al, Astrophys. J 607 (2004) 665 ; S. W. Allen, et al, Mon. Not. R. Astron. Soc. 353 (2004) 457

[14] S. Nesseris, L. Perivolaropoulos, JCAP 0701 (2007) 018

[15] G. Amelino-Camelia, et al, Phys. Rev. D 70, (2004) 107501

[16] J. Maguijo, L. Smolin, Phys. Rev. Lett. 88 (2002) 190403

[17] V. A. Kostelecky and S. Samuel, Phys. Rev. D 39 (1986) 683

[18] P. J. Steinhardt, L. -M. Wang and I. Zlatev, Phys. Rev. D 59 (1999) 123504 
[19] I. Zlatev and P. J. Steinhardt, Phys. Lett. B 459 (1999) 570

[20] H. S. Zhao, A. P. J. Letters 671 (2007) L1

[21] C. G. Bohmer, Eur. Phys. J. C 50 (2007) 423

[22] M. Chaves, D. Singleton, Mod. Phys. Lett. A 22 (2007) 29

[23] H. Wei, R. -G. Cai, JCAP 0709 (2007) 015

[24] H. Wei, Phys. Lett. B 634 (2006) 9

[25] B. Li, D. F. Mota and J. D. Barrow, Phys. Rev. D 77 (2008) 024032

[26] S. D. Sadatian, K. Nozari, work in progress.

[27] A. Vikman, Phys. Rev. D 71 (2005) 023515

[28] M. Libanov, V. Rubakov, E. Papantonopoulos, M. Sami and S. Tsujikawa, JCAP 0708, (2007) 010

[29] O. Bertolami, R. Lehnert, R. Potting, A. Ribeiro, Phys. Rev. D 69 (2004) 083513

[30] B. Feng, X. -L. Wang, X. -M. Zhang, Phys. Lett. B 607 (2005) 35

[31] Y. -f. Cai, H. Li, Y. -S. Piao, X. -m. Zhang, Phys. Lett. B 646 (2007) 141

[32] P. S. Apostolopoulos and N. Tetradis, Phys. Rev. D 74 (2006) 064021 